\title{
Lyman Break Galaxies and Reionization of the Universe
}

\author{
A. Gayler Harford and Nickolay Y. Gnedin \\ Center for Astrophysics and Space Astronomy, University of Colorado, Boulder, CO 80309 \\ gharford@casa.colorado.edu, gnedin@casa.colorado.edu
}

\begin{abstract}
We present the star formation histories, luminosities, colors, mass to light ratios, and halo masses of "galaxies" formed in a simulation of cosmological reionization. We compare these galaxies with Lyman Break Galaxies observed at high redshift. While the simulation is severely limited by the small box size, the simulated galaxies do appear as fainter cousins of the observed ones. They have similar colors, and both simulated and observed galaxies can be fitted with the same luminosity function. This is significant to the process of reionization since in the simulation the stars alone are responsible for reionization at a redshift consistent with that of the GunnPeterson trough seen in the absorption spectra of QSO's. The brightest galaxies at $z=4$ are indeed quite young in accord with observational studies. But these brightest galaxies are free riders - they contributed only about $25 \%$ to the reionization of the universe at $z>6$. Instead, the bulk of the work was done by dimmer galaxies, those that fall within the $28<I<30$ magnitude range at $z \sim 4$. These dimmer galaxies are not necessarily less massive than the brightest ones.

Subject headings: cosmology: theory - cosmology: large-scale structure of universe - galaxies: evolution - galaxies: formation - galaxies: high-redshift - galaxies: starburst - galaxies: stellar content - infrared: galaxies
\end{abstract}

\section{Introduction}

The most dramatic global transition in the recent history of the universe is the reionization of the intergalactic medium. The recent identification of the predicted Gunn-Peterson trough in the absorption spectra of several QSO's has placed this event at about a redshift of 6 (Djorgovski et al. 2002; Becker et al. 2001).

Ionizing radiation from newly formed stars has been suggested as the most likely source of this radiation. Bright, optically selected QSO's appear to be too sparse during the relevant period (Fan et al. 2001). Low brightness AGN's remain a possibility, but no other strong candidates have emerged.

It is natural to look for evidence for these ionizing stars in the accumulating observational data on high redshift $(z \lesssim 4)$ galaxies (Madau et al. 1996; Madau, Pozzetti, \& Dickinson 1998; Steidel et al. 1999; Ouchi et al. 2001; Ferguson, Dickinson, \& Papovich 2002; Hu et al. 2002; Malho- tra \& Rhoads 2002; Lehnert \& Bremer 2003; Kodaira et al. 2003; Iwata et al. 2003). Some studies on Lyman Break Galaxies (LBG) have questioned whether enough stars could have been formed to accomplish reionization at so early a time (Ferguson, Dickinson, \& Papovich 2002; Dickinson et al. 2003).

In the popular Lambda-CDM cosmological model the seeds of galaxy formation lie in minute density fluctuations, which manifest themselves as temperature fluctuations in the Cosmic Microwave Background (CMB). As the fluctuations continue to grow into dark matter halos, they trap gas within their potential wells. That part of the gas that is able to radiate its energy efficiently eventually condenses into dense molecular clouds, that give birth to stars.

Reionization has been studied within this paradigm both by semi-analytical methods (Giroux \& Shapiro 1996; Ciardi \& Ferrara 1997; Haiman \& Loeb 1997, 1998; Madau et al. 1999; Valageas \& 
Silk 1999; Chiu \& Ostriker 2000; Miralda-Escude, Haehnelt \& Rees 2000; Barkana \& Loeb 2000; Oh et al. 2001; Wyithe \& Loeb 2003) and by numerical simulations (Ostriker \& Gnedin 1997; Gnedin \& Ostriker 1998; Gnedin 2000; Nakamoto, Umemura, \& Susa 2001; Razoumov et al. 2002). The former necessarily involve broad simplifying assumptions and, therefore, have limited predictive power. The latter, although more realistic and fully self-consistent, are limited by the dynamical range that can be accommodated by existing numerical techniques on modern supercomputers. The two approaches are complementary and will have to remain so until the next generation of cosmological numerical codes become available on faster computers.

For this paper we use a simulation that was reported in Gnedin (2000). The simulation includes dark matter, gas, star formation, chemistry and ionization balance in the primordial plasma, and an approximate implementation of 3D radiative transfer. All galaxies with total masses in excess of $3 \times 10^{8} \mathrm{M}_{\odot}$ are resolved both spatially and by mass. The simulation was continued until $z=4.0$.

The overall picture for the evolution of the early universe that has emerged from this simulation is as follows:

Star formation first begins in earnest at about $200 \mathrm{Myr}(z \sim 20)$. The overall star formation rate increases to a maximum at about 1 Gyr $(z \sim 5.5)$ and decreases slightly thereafter.

Reionization of the intergalactic medium occurs over a prolonged period of time from about $350 \mathrm{Myr}(z \sim 12)$ to about $900 \mathrm{Myr}(z \sim 6)$, with expanding Hı regions in the low density IGM merging at $z \sim 7$. This latter moment of rapid transition is often identified as the "moment of reionization". This moment approximately corresponds to the mass (volume) averaged neutral fraction in the low density IGM falling below 1 (0.1) percent respectively.

As a result of reionization, the gas content of halos less massive than about $3 \times 10^{9} \mathrm{M}_{\odot}$ solar masses is drastically reduced.

Since this simulation does not include AGN's, it provides a detailed example of how stars alone might be sufficient to accomplish reionization. The addition of AGN's to this scenario would presumably lead to even more ionizing photons, which could take up the slack in the event that the amount of star formation in the simulation were overestimated.

The simulation provides us with a detailed star formation history for each galaxy and thus allows us to make detailed photometric predictions. In this paper we present the star formation histories, luminosities, colors, mass to light ratios, and halo masses of the simulated galaxies.

Our simulation, while state of the art, is only barely sufficient for our purpose, because the computational box is so small. This inherent limitation of any simulation limits the number of bright objects that can be modeled, while the observations approach the galaxy hierarchy from the other end, detecting bright galaxies and missing the dim ones. In this sense we can think of observations and simulations as moving toward each other along the galaxy luminosity curve. Although there is not much overlap yet, the junction point has at last been reached, as we show below.

We argue that to the extent comparisons can be made, our predictions are consistent with observations of Lyman Break Galaxies. The varied star formation histories that we see provide the key to understanding why the observations appear to argue that LBG galaxies are too young to have reionized the universe.

\section{Method}

\subsection{Simulation}

The specific simulation we use in this paper is fully described in Gnedin (2000). The simulation was performed using the Softened Lagrangian Hydrodynamics (SLH) code, which includes dark matter, gas, star formation, chemistry and ionization balance in the primordial plasma, and 3D radiative transfer (in an approximate implementation). All these physical ingredients are required to properly model the process of cosmological reionization.

The simulation of a representative $\mathrm{CDM}+\Lambda$ cosmological model ${ }^{1}$ was performed in a comoving box with the size of $4 h^{-1} \mathrm{Mpc}$ with the total

\footnotetext{
${ }^{1}$ With the following cosmological parameters: $\Omega_{0}=0.3$, $\Omega_{\Lambda}=0.7, h=0.7, \Omega_{b}=0.04, n=1, \sigma_{8}=0.91$, where the amplitude and the slope of the primordial spectrum are fixed by the COBE and cluster-scale normalizations.
} 
mass resolution of $4 \times 10^{6} \mathrm{M}_{\odot}$ and the comoving spatial resolution of $1 h^{-1} \mathrm{kpc}$. This resolution is a reasonable compromise between the need to have the box large enough to accommodate several HII regions and the need to have spatial resolution high enough to resolve sources of ionizing radiation. Convergence studies presented in Gnedin (2000) demonstrate that, while still limited, the resolution of this simulation is sufficient to model reionization at a semi-qualitative level (with precision of the order of $50 \%$ or so).

The simulation was stopped at $z=4$ because at this time the rms density fluctuation in the computational box is about 0.25 , and at later times the box ceases to be a representative region of the universe even for the dark matter.

The simulation was designed in such a way as to approximately reproduce the measured star formation rate density at $z=4$ of about $0.1 \mathrm{M}_{\odot} / \mathrm{yr} / \mathrm{Mpc}^{3}$ (Steidel et al. 1999).

Our simulation assumes that all cosmological reionization occurs via radiation from stellar sources, with star formation parameterized by the phenomenological Schmidt law as discussed in Gnedin (2000a). An alternative and complementary scenario would be one in which the bulk of reionization is produced by Active Galactic Nuclei (AGN). In light of recent high-redshift quasar counts (e.g., Fan et al. 2001), a scenario in which the universe is reionized by bright optically selected QSOs seems unlikely in any event. There remains however the possibility that low brightness AGNs contributed significantly (if not dominantly) to the reionization of the universe.

For the purpose of this paper, however, this simulation is quite suitable for the following reason. Our main goal in this paper is to investigate whether galaxies are capable of reionizing the universe and still appear as young as they do at $z \sim 4$. Thus, by ignoring the AGN component, we consider the most extreme case, since we require galaxies to carry all the burden of reionizing the universe by themselves. One would then expect that if galaxies are capable of reionizing the universe and appear as young as they do at $z \sim 4$, then it will be even easier for the models to agree with observations if some ionization is done by AGNs.

We identify galaxies in the simulation with gravitationally bound objects, which are selected with the DENMAX algorithm of Bertschinger \& Gelb (1991).

\subsection{Population Synthesis}

Population synthesis is carried out using the Starburst99 package (Leitherer et al. 1999). The version we use included the July 2002 update, which revised the UV spectra for $\mathrm{O}$ and $\mathrm{B}$ stars. These spectra, based on the recent work of Smith, Norris, \& Crawther (2002), have decreased ionizing radiation during the early stages of star formation. These changes affect not only the amount of Lyman alpha continuum, but also the amount of Lyman alpha that can be produced if this UV is absorbed by local gas. We choose the instantaneous burst option. This is appropriate because we apply spectral synthesis to individual stellar particles formed in the simulation, which sample the stellar distribution function at discrete time steps.

We choose the lowest metallicity option $(5 \%$ solar) available in Starburst99, since the simulated galaxies have comparable metallicities. Starburst99 maintains same metallicity for each subsequent generation of stars. This simplification should be sufficient for times less than about $1 \mathrm{Gyr}$ (Leitherer et al. 1999).

Ultra-violet absorption by the IGM material along the line of sight (i.e. by the Lyman alpha forest) is taken into account using the method of Madau (1995).

Reddening corrections are made by the method of Calzetti (1997), including the recent calibration data of Leitherer et al. (2002) We find it sufficient to consider a range of reddening corresponding to a color excess of $E(B-V)=0-0.3$. Higher amounts of reddening produce colors inconsistent with nearly all the observed galaxies. This range is in line with the estimate of Shapely et al. (2001) of an average color excess of $E(B-V)=0.15$ for a sample of Lyman break galaxies at a redshift of about 3 . This range is also generally consistent with the results of others for high redshift galaxies.

We also allow for the partial escape of ionizing photons from the modeled galaxies by reducing the flux above $13.6 \mathrm{eV}$ by a factor $f_{\mathrm{ESC}}$, and converting $2 / 3$ of the removed photons into Lymanalpha emission following the recipe of Malhotra \& 




Fig. 1.- Calculated spectrum of a typical bright simulated galaxy at redshift 4.0. Ultra-violet absorption by the Lyman alpha forest has been taken into account. An escape fraction of 1.0 was used with no reddening correction.

Rhoads (2002). The escape fraction $f_{\text {ESC }}$ becomes another parameter in our modeling. We consider three values for this parameter: $0.1,0.5$, and 1.0.

The $A B$ magnitude system is used throughout. We adopt the same cosmology as used in the simulation (flat concordance cosmology with $H_{0}=70 \mathrm{~km} / \mathrm{s} / \mathrm{Mpc}$.

For comparison purposes the $G-I$ colors for observed galaxies over a range of redshifts were corrected to a redshift of 4.0. This was done as follows. For each of a range of $z$ values, including 4.0, a series of 199 model galaxies of different ages was constructed at $5 \mathrm{Myr}$ intervals, each having a single burst of star formation. For each observed galaxy the set of models at the nearest $z$ value was selected. The two bracketing models at this redshift plus the two corresponding models at redshift 4.0 were used to obtain a corrected color using a linear interpolation. This method was feasible because the relationship between $G-I$ and age is, with few exceptions at these intervals, monotonic. A similar correction for $\mathcal{R}-I$ was not possible.

Figure 1 shows an illustrative spectrum of a typical bright galaxy from our simulation. Sharp absorption edges due to intervening Lyman- $\alpha$ and Lyman- $\beta$ forests are clearly visible.



Fig. 2.- Luminosity Function at rest frame 1600 A for simulated galaxies at $z=4.0$ in the $I$ band. Squares show the unreddened luminosity function, while triangles give the luminosity function with $E(B-V)=0.15$ reddening included. Vertical error-bars are 1-sigma Poisson errors. Diamonds show the observed luminosity function of HDF galaxies from Madau et al. (1996), while crosses and asterisks mark the observed LBG luminosity functions at $z \sim 4$ and $z \sim 3$ respectively from Steidel et al. (1999). The dashed line is our Schechter function fit to the combined luminosity function.

\section{Results}

Figure 2 shows a computed luminosity function for the simulated galaxies at $z=4.0$. As a measure of luminosity we use the $I$ band $A B$ magnitude, which corresponds to a rest frame wavelength of about $1600 \mathrm{~A}$. Also shown for comparison is the observed luminosity function for Lyman Break Galaxies at $z \sim 3-4$ from a variety of authors (Madau et al. 1996; Steidel et al. 1999). Because of the difficulties in observing at these redshifts, Steidel et al. (1999) have not attempted to make a Schechter fit at $z=4$, but instead have observed that the data are well described by the Schechter function fitted to Lyman Break Galaxies at a redshift of about 3. This fit, however, does not match the slope of the luminosity function of simulated galaxies at faint magnitudes, but the combined luminosity function (simulated plus observed) can be fit by a single Schechter function with a shallower slope. The parameters of both fits are listed in Table 1. 
Because of the small size of the simulation box, only a few of the observed points are expected to overlap the simulated ones, and these are all from the Hubble Deep Field North, which probed fainter magnitudes than did the ground based observations of Steidel et al. (1999). But it appears that both the simulation and the observations can be fitted with the same luminosity function, implying that the simulation produces galaxies similar to the real ones. The limitation of the small box size directly translates into the upper limit on galaxy luminosities (there should be at least a few galaxies of luminosity $L$ in the simulation box to determine $\phi(L))$. As one can see, our simulation has just enough volume to reach the luminosities of HDF galaxies, and is a factor of 2 too small to have a single galaxy as large as those found by Steidel et al. (1999) in the simulation volume. Thus, we are not able to make comparisons on a galaxy-by-galaxy basis yet, but future simulation will be able to model volumes comparable to those covered by observations in only a few years.

Given a Schechter function fit, we can integrate the total luminosity function to estimate the fraction of starlight in observed and simulated galaxies. We find that the observed galaxies contain about $2 / 3$ of the total luminosity density, while the simulated ones account for about $1 / 3$. The contribution of observed galaxies to reionization is expected to be somewhat smaller however (Gnedin $2000 \mathrm{~b}$ ). This is because these galaxies tend to be clustered (Steidel et al. 1999; Adelberger et al. 1998), and most of the time several of them sit inside the same HiI region. When an HII region grows to be larger than the mean free path of ionizing photons, these photons are wasted.

These arguments imply that the redshift of reionization in the simulation is an underestimate, but not by a large factor because the star formation rate in the simulation increases rapidly with time (Gnedin 2000a). If we assume that the simulation underestimates the volume filling fraction of the ionized gas at any given time by, say, $50 \%$,

Table 1: Schechter function fit parameters

\begin{tabular}{ccc}
\hline \hline Parameter & Steidel et al. fit & Our fit \\
\hline$\alpha$ & -1.60 & -1.22 \\
$M_{*}$ & -20.9 & -20.4 \\
$\phi_{*}\left(\mathrm{Mpc}^{-3}\right)$ & $1.8 \times 10^{-2}$ & $5.7 \times 10^{-2}$ \\
\hline
\end{tabular}

then the redshift of reionization is underestimated in the simulation by about $\Delta z \sim 1.5$. As we emphasized above, our simulation is clearly insufficient to give a quantitatively accurate model of reionization, so this level of precision is more than acceptable for our purposes.

A remarkable feature of Fig. 2 is flattening of the luminosity function in the dwarf galaxy range. This effect is the result of the inhibited accretion of gas onto low mass halos because of photoionization, sometimes improperly called "photoevaporation" (Thoul \& Weinberg 1996; Quinn, Katz, \& Efstathiou 1996; Weinberg, Hernquist, \& Katz 1997; Navarro \& Steinmetz 1997; Gnedin 2000b; Chiu, Gnedin, \& Ostriker 2000; Sommerville 2002; Benson et al. 2002a,b), and has been reproduced by earlier simulations (Nagamine 2002).

Figure 3 shows Color-Magnitude Diagrams (CMD) for the simulated and observed galaxies. Because the simulation cannot predict the redenning correction, we plot each simulated galaxy as a dotted region (which appears almost like a single line), spanning a range in redenning corrections from $E(B-V)=0$ to $E(B-V)=0.3$, consistent with values found by Steidel et al. (1999), and a range of escape fractions from $f_{\mathrm{ESC}}=0.1$ to $f_{\mathrm{ESC}}=1.0$.

One can see that simulated galaxies have similar colors to the observed ones, which suggests that the simulation captures at least some of the physics, which is going on in these galaxies. Let us now imagine a "super-simulation", with so large a box that it can be considered infinite. Because the colors of our brightest galaxies do not change much with magnitude, it is plausible to assume that simulated galaxies in the magnitude range $23<I<25$ would have similar colors to the observed ones. If we plausibly assume that the simulated luminosity function, when extended to higher luminosities, would match the observed one reasonably well, then the total luminosity density in such a "super-simulation" would be a factor of 3 higher than in our simulation, and, thus, the redshift of reionization would be even higher than $z=7$ - the redshift of reionization in our simulation - by about $\Delta z \sim 1.5$. Such a "supersimulation" would serve as a strong counter example to claims that LBGs at $z \sim 4$ are too young to reionize the universe by $z \sim 6$.

Why, then, is observational determination of 

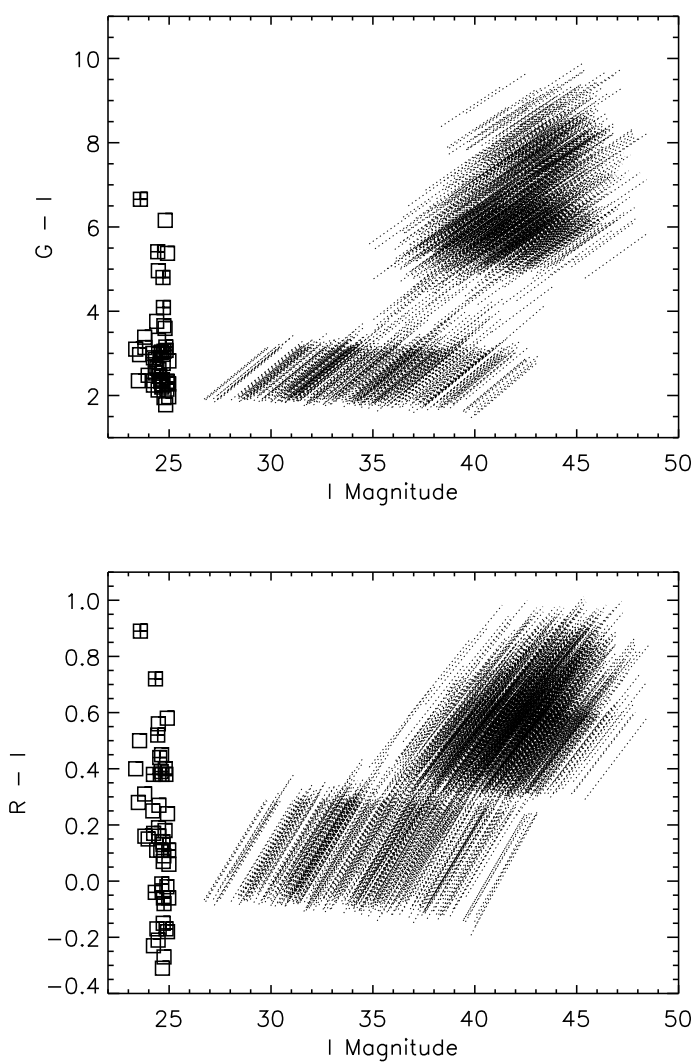

Fig. 3.- Color-Magnitude Diagrams for Simulated Galaxies at $z=4.0$ shown as $(a) G-I$ bv $I$ and $(b) \mathcal{R}-I$ vs $I$. Each galaxy is represented by a region spanning the range of of reddening from $E(B-V)=0$ to $E(B-V)=0.3$ and the range of escape fractions from $f_{\mathrm{ESC}}=0.1$ to $f_{\mathrm{ESC}}=1.0$. Squares are observed LBGs from Steidel et al. (1999) in the redshift range from $z=3.5$ to $z=4.8$. A cross within the square indicates that only a minimum $G$ magnitude could be measured.
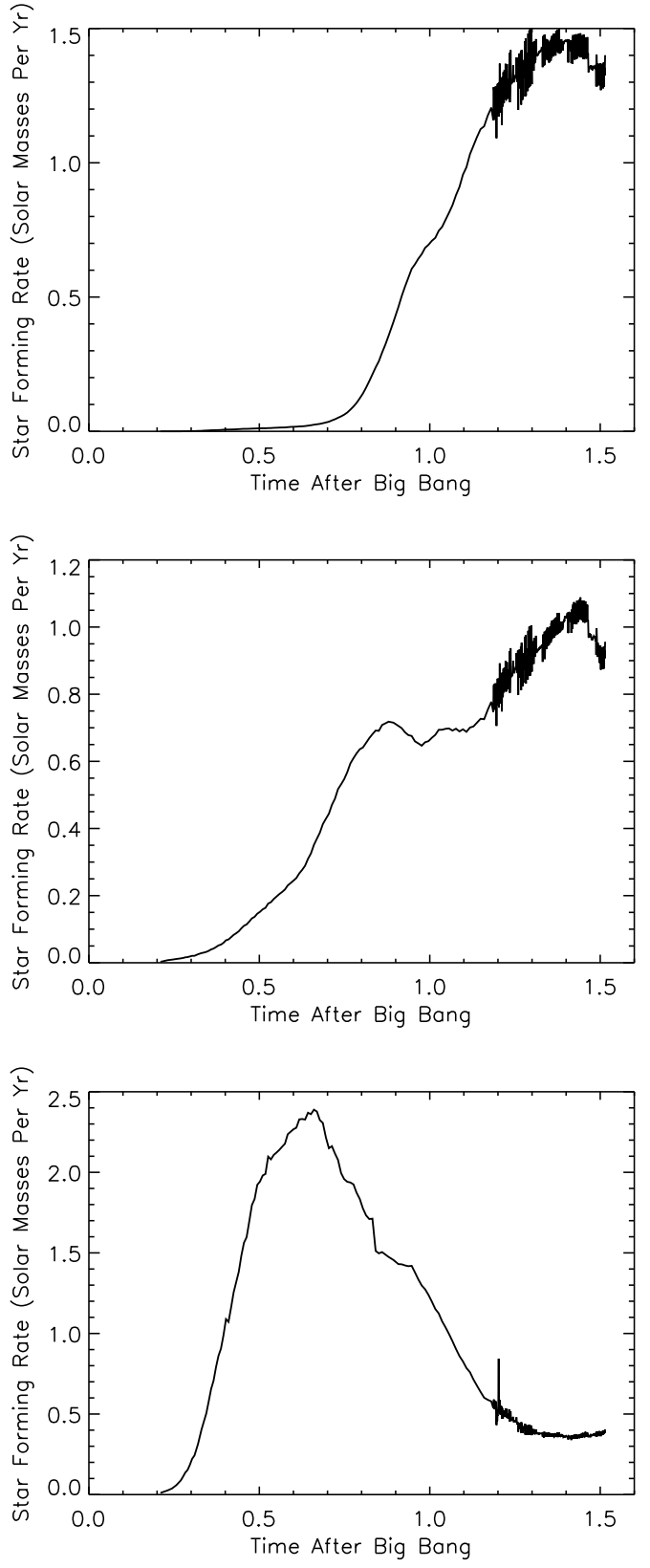

Fig. 4.- Three illustrative star formation histories for simulated galaxies with different luminosities: $I=26.9(a), I=27.2(b), I=28.0(c)$. Despite the very different histories, all three have very similar $G-I$ and $\mathcal{R}-I$ colors. The temporal sampling of star formation histories is 10 times higher after $1.2 \mathrm{Gyr}$, showing fine structure variability on a few Myr time scale due to formation of individual star clusters, which are resolved (by mass) in the simulation. 


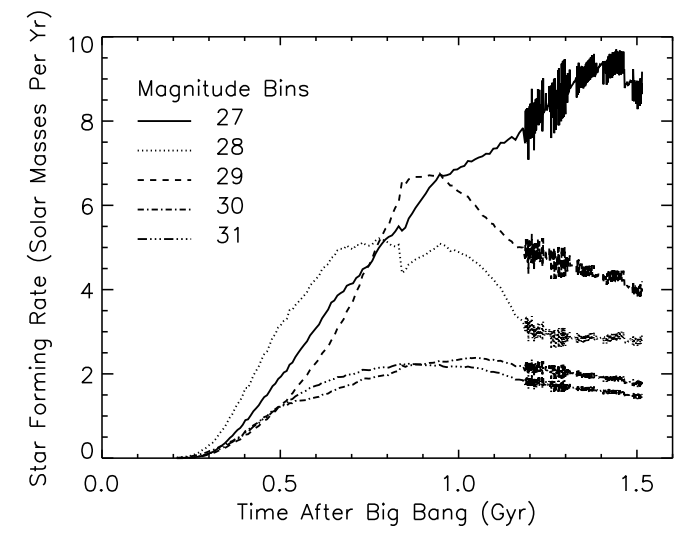

Fig. 5.- Total star formation rates in galaxies that appear within a given magnitude bin at $z=$ 4 as a function of cosmic time. Each indicated magnitude is the central value of a bin of width 1.0 magnitudes.

ages so misleading? Figure 4 illustrates the diversity of star formation histories in simulated galaxies. While our brightest galaxies $(I \lesssim 27$, such as the one shown in panel $a$ ) are experiencing a peak of star formation at around $z=4$, one magnitude dimmer galaxies $(I \gtrsim 28)$ were more luminous in the past - indeed, at the time of reionization (at $z \sim 7$ in this simulation) - and by $z \sim 4$ their star formation rate has decreased by a factor of several. In fact, the galaxy shown in panel $(c)$ has $1.1 \times 10^{9} \mathrm{M}_{\odot}$ in stars, whereas the galaxy in panel (a), while being more than a magnitude brighter, has a stellar mass of only $7 \times 10^{8} \mathrm{M}_{\odot}$. We, therefore, conclude that galaxies that reionized the universe at $z \sim 7$ appear at $z \sim 4$ within the magnitude range of $28 \lesssim I \lesssim 30$, while LBGs that are observed at $z \sim 4$ with $23 \lesssim I \lesssim 25$ are indeed very young, experiencing their first major episode of star formation (we refrain from using the word "burst", because the star formation history of a galaxy shown in panel $a$, while strongly rising by $z \sim 4$, can still be hardly called a "burst").

Figure 5 illustrates this point further. It shows total star formation rates for galaxies that appear within different magnitude bins at $z=4$. In agreement with individual examples from Fig. 4, the star formation rate at $z=4$ is dominated by our brightest galaxies, but these galaxies contribute only about $20 \%$ to the star formation at $z \sim 7$. At that redshift more than half of the total star

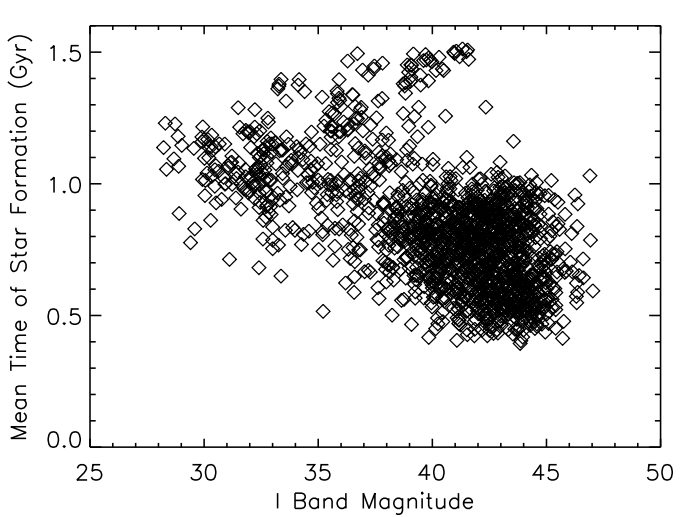

Fig. 6.- Mean star formation time for all simulated galaxies as a function of their magnitudes at $z=4$.

formation rate is contributed by galaxies that appear within the $28<I<30$ magnitude bin at $z \sim 4$.

The large variation in possible star formation histories is further confirmed by Figure 6, which shows mean mass-weighted star formation ages for all simulated galaxies as a function of their magnitudes at $z=4$,

$$
t_{S F}=\frac{1}{M_{*}} \int_{0}^{t_{f}} t \operatorname{SFR}(t) d t
$$

where $M_{*}$ is the total stellar mass. Even for our brightest galaxies the variation is significant, reaching up to a factor of 3 for the dimmest ones. A remarkable feature of Fig. 6 is a large concentration of galaxies with $40<I<45$ and little star formation after 1 Gyr. It is tempting to identify these galaxies with dwarf spheroidals: they are old and would have luminosities of the order of $10^{5-6} L_{\odot}$ at $z=0$. A small fraction of these galaxies still have active star formation at $z \sim 4$, which may explain the diverse star formation histories of dwarf spheroidals in the Local Group (Grebel 1998; Mateo 1998). We leave this as a possible speculation, as this is not the goal of this paper, however.

Observations at longer wavelengths and/or at fainter magnitudes would be more sensitive to older populations of stars. Unfortunately, these observations are very difficult to make at a redshift of 4 , and they will most likely have to wait for JWST and 30-meter class ground telescopes. 


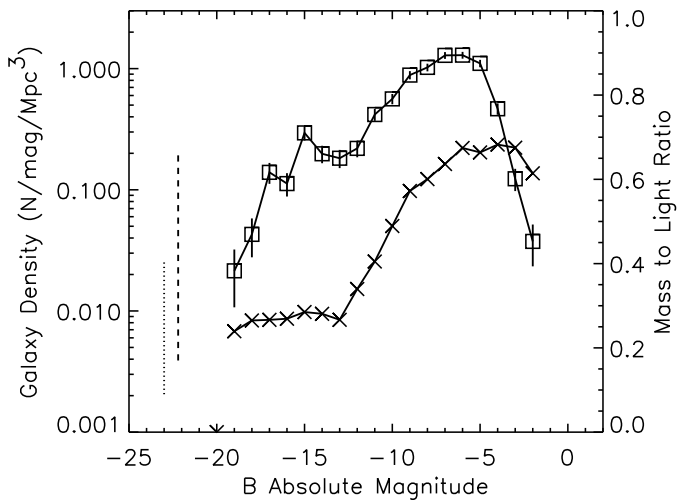

Fig. 7.- Mass-to-light ratio for simulated galaxies at $z=4$. Squares show the luminosity function of the simulated galaxies as would be observed in the $\mathrm{K}$ band with $E(B-V)=0.15$ reddening. Vertical lines through the symbols represent 1-sigma Poisson errors. Crosses show the stellar mass-tolight ratio in solar units computed with the same reddening. The vertical lines on the left show the stellar mass-to-light ranges found by Dickinson et al. (2003) assuming metallicities of $1 / 5$ solar (dotted) and solar (dashed).

But those instruments are on the horizon, and one might hope to indeed observe the culprits of reionization within the 10-year time frame.

Whatever the global history of star formation we would at least expect that the total stellar content of the universe would be monotonic with time. Thus, we have compared the stellar content of the simulated galaxies with the global history of total stellar mass as presented in Dickinson et al. (2003). In Figure 7 we show the B band rest frame luminosity function and mass-to-light ratios of the simulated galaxies, calculated from the $\mathrm{K}$ band magnitudes. Vertical lines give the comparison with Dickinson et al. (2003) estimates. Our results are in comfortable agreement with these estimates for all galaxies brighter than dwarf spheroidal candidates.

Finally, Figure 8 shows cumulative mass and luminosity densities of simulated galaxies as compared with Dickinson et al. (2003) estimates. Again it appears that the simulated galaxies are in a reasonable agreement with the observational data. We would like to emphasize that we are quite comfortable with a factor of 2 agreement:

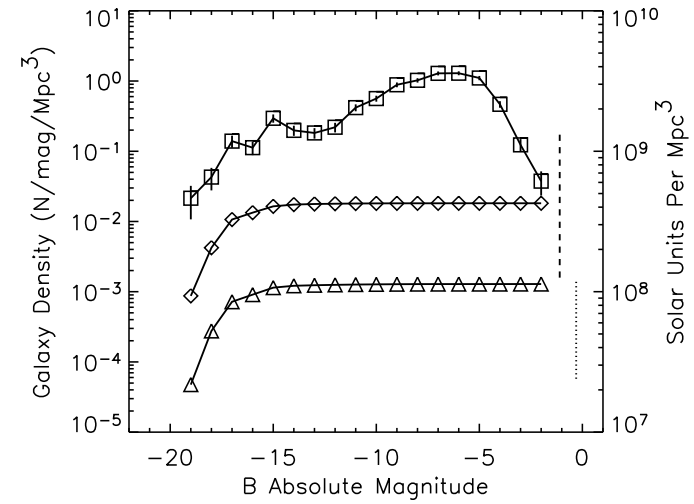

Fig. 8.- Cumulative stellar mass and B band luminosity. Squares show the luminosity function of the simulated galaxies at $z=4$ as would be observed in the $\mathrm{K}$ band. Vertical lines represent 1-sigma Poisson errors. Triangles show the total stellar mass in simulated galaxies integrated from the lowest magnitude to the plotted magnitude. Diamonds trace the luminosity density of simulated galaxies as would be observed in the $\mathrm{K}$ band with $E(B-V)=0.15$ reddening. The dotted line is the mass density range observed by Dickinson et al. (2003). The dashed line is their observed luminosity density range obtained by integrating over a Schechter fit.

one should remember that the simulation can only account for about $1 / 3$ of the total light because of the small box size. In fact, in the imaginary "super-simulation", discussed above, the total stellar mass would be up to a factor of 3 higher (depending on whether the mass-to-light ratio goes down for brighter galaxies) and would not agree with Dickinson et al. (2003). One could hypothesize about a source of this potential discrepancy, including anything from a non-standard IMF to incorrect stellar synthesis models, but for the purpose of this paper we are content with the level of agreement we obtain, given substantial limitations of our simulation.

It would also be interesting to compare the luminosities of Lyman Break Galaxies and their dark matter masses, because the relation of LBGs and their dark matter halos is still poorly understood (Somerville, Primack, \& Faber 2001; Wechsler et al. 2001; Somerville 2002; Benson et al. 2002a,b). In Figure 9 we show this relation. As one can see, 




Fig. 9.- Scatter plot of absolute magnitude at 1600 A versus total (dark matter + gas + stellar) mass of simulated galaxies at redshift 4 . Reddening equivalent to a color excess of 0.15 has been applied as well as extinction due to absorption by the Lyman alpha forest.

Lyman Break Galaxies represent a diverse population. For example, our brightest galaxies with absolute magnitudes between -18 and -17 cover about two magnitudes in the total mass: some of them are the most massive galaxies in the simulations, but some of them have significantly lower masses and are experiencing a starburst at $z=4$. There exists a general trend of less massive galaxies being dimmer, but the scatter around the mean relation is very large.

\section{Discussion}

So, how well does the simulation do? While the computational box of our simulation is clearly insufficient to have even a single galaxy as luminous as those of Steidel et al. (1999), simulated galaxies do appear as fainter cousins of the observed ones. They have similar colors, and simulated and observed galaxies together can be fitted by a single luminosity function.

They are also faring well in their abilities to reionize the universe. Star formation histories of simulated galaxies are diverse, and vary systematically with magnitude. The brightest galaxies at $z=4$ are indeed quite young, in accord with conclusions of Shapely et al. (2001) and Ferguson et al. (2002). But these brightest galaxies contribute only about $25 \%$ of ionizing photons at $z>6$.
The bulk of work of reionizing the universe was done by dimmer galaxies, those that fall within the $28<I<30$ magnitude range at $z \sim 4$, but which are not necessarily less massive than the bright ones.

We expect that detailed simulations will become increasingly important in sorting out the various possibilities. The general picture that our simulation produces is at least consistent with observations of Lyman Break Galaxies, an encouraging message to theorists.

The recent results from the WMAP mission (Kogut et al. 2003) indicate that the optical depth to Thompson scattering is significantly larger than in our simulation. While this measurement cannot pin down the redshift of reionization, it suggests, if correct, that there was a considerable ionizing flux at early times. Our simulation provides no support for a stellar origin of this ionizing flux. Perhaps, very massive population III stars, which we have not considered, could be responsible.

Because, as we have discussed, the photoionization feedback likely plays a significant role in the evolution of faint Lyman Break Galaxies, one might wonder how the WMAP results affect the conclusions of this paper. As has been shown by several previous studies (Gnedin 2000b and references herein), the photoionization feedback results in the substantial loss of the gas mass of objects with circular velocities below about $40 \mathrm{~km} / \mathrm{s}$. However, this characteristic circular velocity is essentially redshift independent. So even if the universe was fully ionized throughout its entire history, the effect of the photoionization feedback at $z=4$ would be essentially at the same level as in our simulations. Our conclusion about a reasonable agreement between the simulation and the data is, in fact, independent of the redshift of reionization as long as it is above about 6 .

We are grateful to Chuck Steidel for providing us with his custom filter shapes and for enlightening comments during his visit to CU. This work was supported by NSF grant AST-0134373. This work was also partially supported by National Computational Science Alliance under grant AST-960015N and utilized the SGI/CRAY Origin 2000 array at the National Center for Supercomputing Applications (NCSA). 


\section{REFERENCES}

Adelberger, K. L., Steidel, C. C., Giavalisco, M., Dickinson, M., Pettini, M., \& Kellogg, M. 1998, ApJ, 505, 18

Barkana, R., \& Loeb, A. 2000, ApJ, 539, 20

Becker, R. H., et al. 2001, AJ, 122, 2850

Benson, A. J., Lacey, C. G., Baugh, C. M., Cole, S., \& Frenk, C. S. 2002a, MNRAS, 333, 156

Benson, A. J., Frenk, C. S., Lacey, C. G., Baugh, C. M., \& Cole, S. 2002b, MNRAS, 333, 177

Bertschinger, E., \& Gelb, J. 1991, J. Comput. Phys., 5, 164

Calzetti, D. 1997, AJ, 113, 162

Chiu, W. A., Gnedin, N. Y., \& Ostriker, J. P. 2000, ApJ, 563, 21

Chiu, W. A., \& Ostriker, J. P. 2000, ApJ, 534, 507

Ciardi, B., Ferrara, A. 1997, ApJ, 483, L5

Dickinson, M., Papovich, C., Ferguson, H. C., \& Budavari, T. 2003, ApJ, in press (astro$\mathrm{ph} / 0212242)$

Djorgovski, S. G., Castro, S., Stern, D., Mahabal, A. A. 2001, ApJ, 560, L5

Fan, X., et al. 2001, AJ, 122, 2833

Ferguson, H. C., Dickinson, M., Papovich, C. 2002, ApJ, 569, L65

Giroux, M. L., \& Shapiro, P. R. 1996, ApJS, 102, 191

Gnedin, N. Y. 2000a, ApJ, 535, 530

Gnedin, N. Y. 2000b, ApJ, 542, 535

Gnedin, N. Y., \& Ostriker, J. P. 1997, ApJ, 486, 581

Grebel, E. 1998, IAUS, 192, 1

Haiman, Z., \& Loeb, A. 1997, ApJ, 483, 21

Haiman, Z., \& Loeb, A. 1998, ApJ, 503, 505

Hu, E. M., Cowie, L. L., McMahon, R. G., Capak, P., Iwamuro, F., Kneib, J.-P., Maihara, T., \& Motohara, K. 2002, ApJ, 568, L75

Iwata, I., Ohta, K., Tamura, N., Ando, M., Wada, S., Watanabe, C., Akiyama, M., \& Aoki, K. 2003, PASJ, in submitted (astro-ph/0301084)

Kodaira, K., et al. 2003, ApJ, submitted (astro$\mathrm{ph} / 0301096$ )
Kogut, A., et al. 2003, ApJ, in press (astro$\mathrm{ph} / 0302213)$

Lehnert, M. D., \& Bremer, M. 2003, ApJ, submitted (astro-ph/0212431)

Leitherer, C., Schaerer, D., Goldader, J. D., Gonzalez Delgado, R. M., Robert, C., Foo Kune, D., de Mello, D. F., Devost, D., \& Heckman, T. M. 1999, ApJS, 123, 3

Leitherer, C., Li, I.-H., Calzetti, D., \& Heckman, T. M. 2002, ApJS, 140, 303

Madau, P. 1995, ApJ, 441, 18

Madau, P., Ferguson, H. C., Dickinson, M., Giavalisco, M., Steidel, C. C., \& Fruchter, A. 1996, MNRAS, 283, 1388

Madau, P., Haardt, F., \& Rees, M. J. 1999, ApJ, 514,648

Madau, P., Pozzetti, L., \& Dickinson, M. E. 1998, ApJ, 498, 106

Malhotra, S., \& Rhoads, J. E. 2002, ApJ, L71

Mateo, M. 1998, ARA\&A, 36, 435

Miralda-Escudé, J., Haehnelt, M., \& Rees, M. J. 2000, ApJ, 530, 1

Nagamine, K. 2002, ApJ, 564, 73

Nakamoto, T., Umemura, M., \& Susa, H. 2001, MNRAS, 321, 593

Navarro, J., \& Steinmetz, M. 1997, ApJ, 478, 13

Oh, S. P., et al. 2001, ApJ, 562, L1O

Ostriker, J. P., \& Gnedin, N. Y. 1996, ApJ, 472, L63

Ouchi, M., Shimadaku, K., Okamura, S., Doi, M., Furusawa, H., Hamabe, M., Kimura, M., Komiyama, Y. et al. 2001, ApJ, 558, L83

Quinn, T., Katz, N., \& Efstathiou, G. 1996, ApJ, $278,49 \mathrm{P}$

Razoumov, A. O., Norman, M. L., Abel, T., \& Scott, D. 2002, ApJ, 572, 695

Shapley, A. E., Steidel, C. C., Adelberger, K. L., Dickinson, M., Giavalisco, M., \& Pettini, M. 2001, ApJ, 562, 95

Smith, L. J., Norris, R. P. F., \& Crawther, P. A. 2002, MNRAS, 337, 1309

Somerville, R. S. 2002, ApJ, 572, L23

Somerville, R. S., Primack, J. R., \& Faber, S. M. 2001, MNRAS, 320, 504 
Steidel, C. C., Adelberger, K. L., Dickinson, M., Giavalisco, M., Pettini, M., \& Kellogg, M. 1998, ApJ, 492, 428

Steidel, C. C., Adelberger, K. L., Giavalisco, M., Dickinson, M., \& Pettini, M. 1999, ApJ, 519, 1

Thoul, A. A., \& Weinberg, D. H. 1996, ApJ, 465, 608

Valageas, P., \& Silk, J. 1999, A\&A, 347, 1

Wechsler, R. H., Somerville, R. S., Bullock, J. S., Kolatt, T. S., Primack, J. R., Blumenthal, G. R., \& Dekel, A. 2001, ApJ, 554, 85

Weinberg, D. H., Hernquist, L., \& Katz, N. 1997, ApJ, 477, 8

Wyithe, S., \& Loeb, A. 2003, ApJ, in press (astro$\mathrm{ph} / 0209056$ )

This 2-column preprint was prepared with the AAS $\mathrm{LAT}_{\mathrm{E}} \mathrm{X}$ macros v5.0. 